\title{
Matrix cracks around fibre breaks and their effect on stress redistribution and failure development in unidirectional composites
}

\author{
Yentl Swolfs* ${ }^{* 1}$, Robert M. McMeeking ${ }^{2,3,4}$, Ignaas Verpoest ${ }^{1}$, Larissa Gorbatikh ${ }^{1}$ \\ ${ }^{1}$ Department of Materials Engineering, KU Leuven, Kasteelpark Arenberg 44 bus \\ 2450, Belgium \\ ${ }^{2}$ Department of Mechanical Engineering, University of California, Santa Barbara, CA \\ 93106, USA \\ ${ }^{3}$ Materials Department, University of California, Santa Barbara, CA 93106, USA \\ ${ }^{4}$ School of Engineering, University of Aberdeen, King's College, Aberdeen AB24 \\ 3UE, Scotland, UK. \\ *Corresponding author: Y. Swolfs (yentl.swolfs@mtm.kuleuven.be)
}

\begin{abstract}
Despite the crucial significance of failure prediction in composites, such an objective remains challenging, even in unidirectional (UD) systems. A strength model for UD composites was used that has great versatility in handling various matrix and fibre behaviours. This model includes a simplified superposition principle that was found to be reliable in predicting stress concentration factors irrespective of the presence of matrix cracks. The model revealed the negligible influence of matrix cracks on stress concentrations, ineffective length, cluster development and failure strain. The presence of matrix cracks can therefore be safely neglected in models for UD composites. This information is important for experimental validations and for advancing the state of the art in strength models for UD composites.
\end{abstract}

Keywords: A. Polymer-matrix composites (PMCs); B. Matrix cracking; C. Stress concentrations; C. Probabilistic methods

\section{Introduction}

Failure of fibre-reinforced composites often occurs suddenly and without any visible signs of damage serving as a warning. This feature is caused by the gradual development of microscopic damage. Predicting such damage is challenging, especially in multidirectional composites. In most cases, however, the failure of multidirectional composites coincides with the failure of the fibres oriented in the loading direction. Hence, understanding the $0^{\circ}$ tensile failure of unidirectional (UD) composites is vital.

Monotonic loading of a UD composite results in stochastic fibre failure [1], with their failure strength typically obeying a Weibull distribution. Each break locally causes the fibre to lose its load transfer capability and shed its load to nearby fibres $[2,3]$. The fibres nearby are hence subjected to stress concentrations, and their failure probability increases. As the matrix surrounding the fibre break is loaded in shear, stress is transferred back into the broken fibre. At a characteristic distance from the fibre break plane, the stresses in both the broken and intact fibres return to their nominal value. The increased failure probability of the nearby fibres causes the development of break clusters [4]. These clusters grow with increasing strain, until one of them reaches the 
critical cluster size. This cluster then propagates unstably and leads to the final failure of the composite.

Failure development is dominated by two features: (1) the Weibull distribution of fibre strength and (2) the stress redistribution around a fibre break. The Weibull strength distribution determines the stochastic sequence of fibre failure. Many Weibull strength distributions have been characterised experimentally, but the results can be quite different, even for a given fibre type. For example, this is the case for T300 fibres $[5,6]$ and T800 carbon fibres $[7,8]$.

Stress redistributions around single fibre breaks have been investigated extensively by shear-lag analysis [9-12] and by the finite element (FE) method [13-18]. The two vital parameters in UD composite strength predictions are the stress concentration factors (SCF) in the intact fibres and the ineffective length in the broken fibre. Both parameters are strongly influenced by the modelling assumptions. The importance of the matrix, both for normal and shear stress transfer and through its inelastic behaviour, has been demonstrated by many authors [16,19-22]. In addition, random fibre packings introduce variations in the SCFs, whereas they are deterministic for regular packings [13,23-25].

Another vital assumption concerns the stress singularity around a fibre break. Stress concentrations in the matrix around a fibre break are infinite for elastic, well-bonded materials. The matrix and interface are thus unlikely to be able to cope with this, yet many models assume perfect bonding and an intact matrix [15,26,27]. Three scenarios or combinations thereof can occur: (1) the matrix yields [16,19-22], (2) the fibre-matrix interface debonds $[21,24,28]$, or (3) the matrix cracks in the fibre break plane [29-32]. Matrix yielding is typical in thermoplastic polymer matrix composites, and can also occur in thermosets. Fibre-matrix debonding typically occurs for weak interfacial bonds, and is common in ceramic matrix composites. Matrix yielding and fibre-matrix debonding have been extensively investigated [16,19-22,24,28], but studies on matrix cracking remain scarce.

Matrix cracks are commonplace in ceramic matrix composites [33], but mainly as a phenomenon occurring prior to fibre failure. In polymer matrix composites however, both phenomena are interlinked. Several authors have observed matrix cracking around fibre breaks during single fibre fragmentation tests [29-32]. This occurred typically in material systems with a strong fibre/matrix interfacial bond and a low stiffness [32]. Including matrix cracking in strength models for polymer matrix composites has lagged behind. Li et al. [34] and Mishnaevsky and Brøndsted [35] developed models involving matrix cracks around fibre breaks, but the influence of these cracks on stress redistribution and strength of the composite was not investigated yet.

While matrix cracks around fibre breaks are neglected by most strength models, experiments have indicated that they sometimes occur in UD composites [29-32]. The purpose of the present paper is to analyse the influence of matrix cracks could have on the strength and damage development in UD composites. Their influence on the stress redistribution around fibre breaks and on composite failure development is explored. 


\section{Stress redistributions around fibre breaks}

\subsection{FE model for stress redistribution}

A vital aspect of every strength model for UD composites is an accurate description of the stress redistribution caused by a single fibre break. The FE model and its input parameters have been extensively described in Swolfs et al. [13,14]. The main difference is that an elasto-plastic epoxy matrix was used instead of an elastic one. The data from Okabe et al. [6] was used for this purpose.

A single fibre break is surrounded by intact fibres in a random fibre packing. A displacement is applied to the bottom surface of the model (see Fig. 1), corresponding to an applied strain of $2 \%$. Symmetry conditions are applied to the entire top surface, but not to the middle fibre, representing the broken status of that fibre. Matrix cracks around fibre breaks are simulated in a similar fashion, by eliminating the symmetry condition from the corresponding matrix crack area. Models without matrix cracks were simulated using a baseline and an improved strategy. The difference between both strategies lies in the boundary conditions applied to nodes at the perimeter of the fibre break. The baseline strategy applied symmetry conditions to these nodes, as this most closely reflects the assumption that matrix cracks do not occur. The improved strategy however, released these nodes, which is equivalent to a tiny matrix crack with a width of just $13 \mathrm{~nm}$.

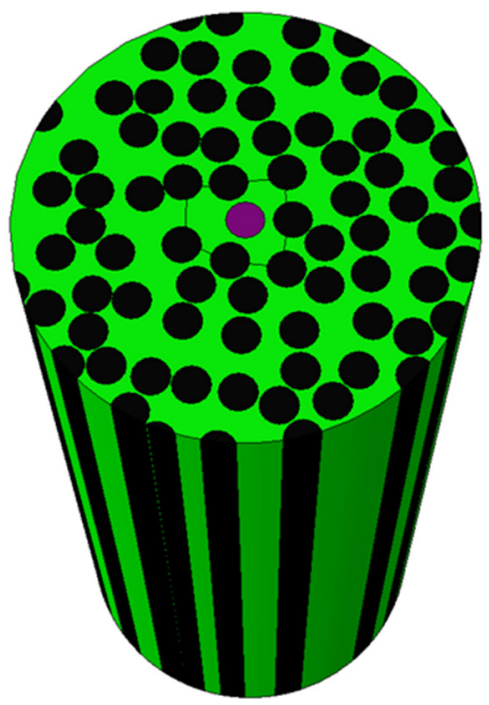

(a)

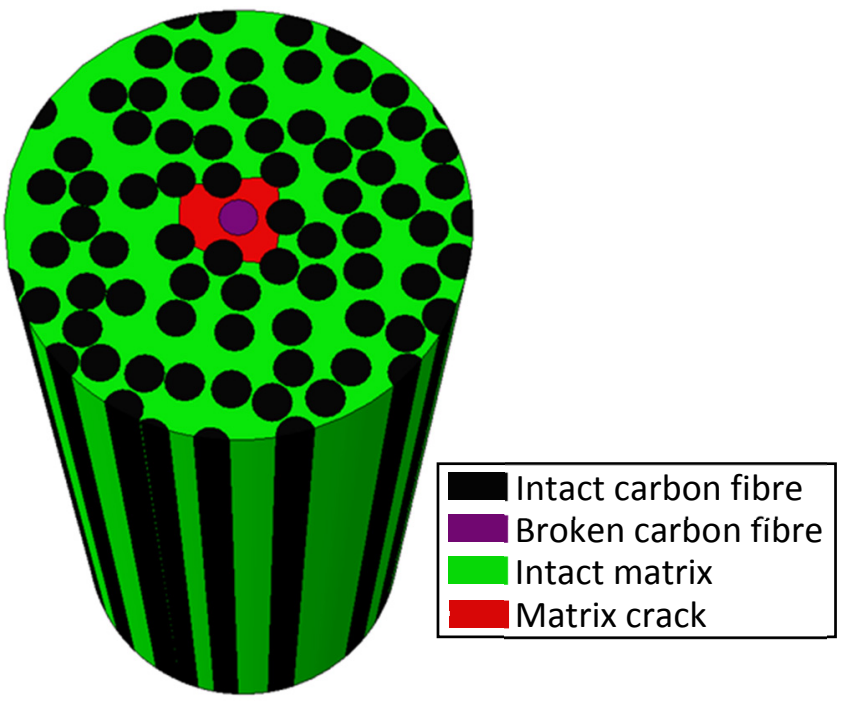

(b)

Figure 1: A 3D view of the FE model of a single fibre break: (a) without a matrix crack, and (b) with a medium-sized matrix crack.

The SCFs are calculated by averaging the stress over the cross-section of the fibres and dividing this average by the far field stress. The SCFs will always be expressed as the percentage by which they exceed unity.

Five FE models were made for each of the three types of boundary conditions. The models without and with a matrix crack are illustrated in Fig. 1. In the absence of experimental data on the size of the matrix crack, the matrix crack is assumed to be constrained by nearby fibres. Since that defines uniquely neither the size nor the shape of the matrix crack, a sensitivity analysis is performed to assess its influence. Three 
different matrix crack sizes with an area of $60 \mu \mathrm{m}^{2}, 150 \mu \mathrm{m}^{2}$ and $205 \mu \mathrm{m}^{2}$ were implemented in one specific fibre packing. The SCFs and ineffective length were found to be fairly insensitive to the shapes and sizes of the matrix crack. A relative difference of only $12 \%$ is found for the maximum SCFs when comparing the smallest and largest crack models. The difference in ineffective length is smaller than 1\%. A medium-sized matrix crack will therefore be used in the assessment of SCFs. The area of the matrix crack varies between 100 and $155 \mu \mathrm{m}^{2}$ depending on the packing. An example of such a crack was shown in Fig. 1b.

To analyse interactions among fibre breaks, FE models with multiple fibre breaks are created. These fibre breaks are located in the same plane, as this situation has the strongest amplification of the SCFs.

\subsection{Non-interacting breaks}

The primary influence of the matrix cracks occurs through a change in the stress redistribution around fibre breaks. This section analyses this redistribution in the broken and intact fibres, for the case of a non-interacting or single fibre break.

Fig. 2 depicts stress recovery profiles in the broken fibre. Fibre stress is plotted as a percentage of the nominal level caused by the global axial strain. The profile for the baseline solution without matrix cracks seems to start at $45 \%$, while the two other ones start at the origin. Since the entire surface of the fibre break is traction-free, the average longitudinal fibre stress is actually zero in the fibre break plane. In the baseline solution, however, the perimeter of the fibre break is constrained. This constraint leads to large stresses on the perimeter of the fibre break and hence a rapid stress build-up to $45 \%$ within the first layer of elements. This is not visible in Fig. 2, as this layer has a thickness of only 0.02 times the fibre radius. A similar stress build up was also reported in Fiedler et al. [36], although they reported a smaller effect. Further mesh refinements had only a minor influence on the stress singularity. This stress singularity cannot occur physically and should be relieved through some mechanism, of which matrix cracks is one possibility. The baseline and improved solution strongly differ. We argue that the boundary conditions used in the improved solution are more appropriate for modelling a fibre break without matrix crack. This strategy yields a more realistic SCF profile in comparison with Raman spectroscopy data [37].

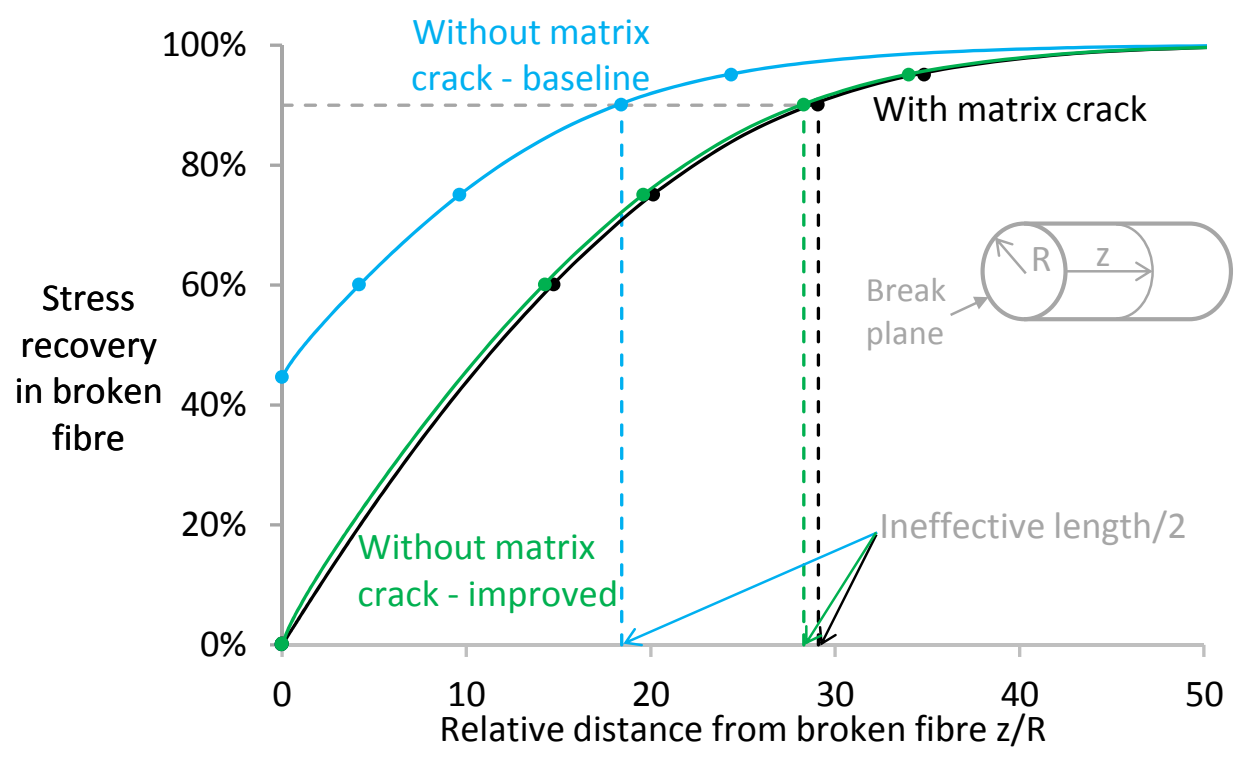


Figure 2: Representative stress recovery profiles in fibres with a single break. The dashed lines indicate half of the ineffective length, while the dots indicate the five reference points that are used for the strength model to characterise stress recovery in the fibre.

The ineffective length is defined as the length over which the broken fibre recovers $90 \%$ of the nominal stress. The ineffective length for baseline models without matrix cracks is only $37 \pm 2$ times the fibre radius. This is significantly shorter than the $57 \pm 3$ and 58 \pm 4 times the fibre radius for improved models without a matrix crack and models with a matrix crack respectively.

Five reference points are used to transfer this information into the strength model: the point in the first layer of elements, and the points where $60 \%, 75 \%, 90 \%$ and $95 \%$ of the stress are recovered, see Fig. 2. The strength model uses piecewise linear interpolation to characterise the entire stress recovery profile.

Fig. 3 depicts the influence of matrix cracks on the maximum SCFs in the intact fibres around the fibre break, defined as the percentage by which it exceeds unity. The addition of matrix cracks doubles the SCF compared to baseline models without matrix cracks. The improved solution results in SCFs that are slightly lower than for models with a matrix crack.

Fig. 3 only presents the maximum SCF values along the fibre, while the strength model requires the entire SCF profile along the intact fibres. Linear or logarithmic trend line formulae are computed for 4 reference points (see Fig. 4) as a function of the relative distance from the crack plane. Fig. 3 shows an example of this procedure for the first point, namely the maximum SCF. The trend line equations are entered into the strength model. Piecewise linear interpolation is used to compute the value at every point along the profile. In this way, the accuracy of the FE approach is exploited without requiring FE simulations during the strength model.

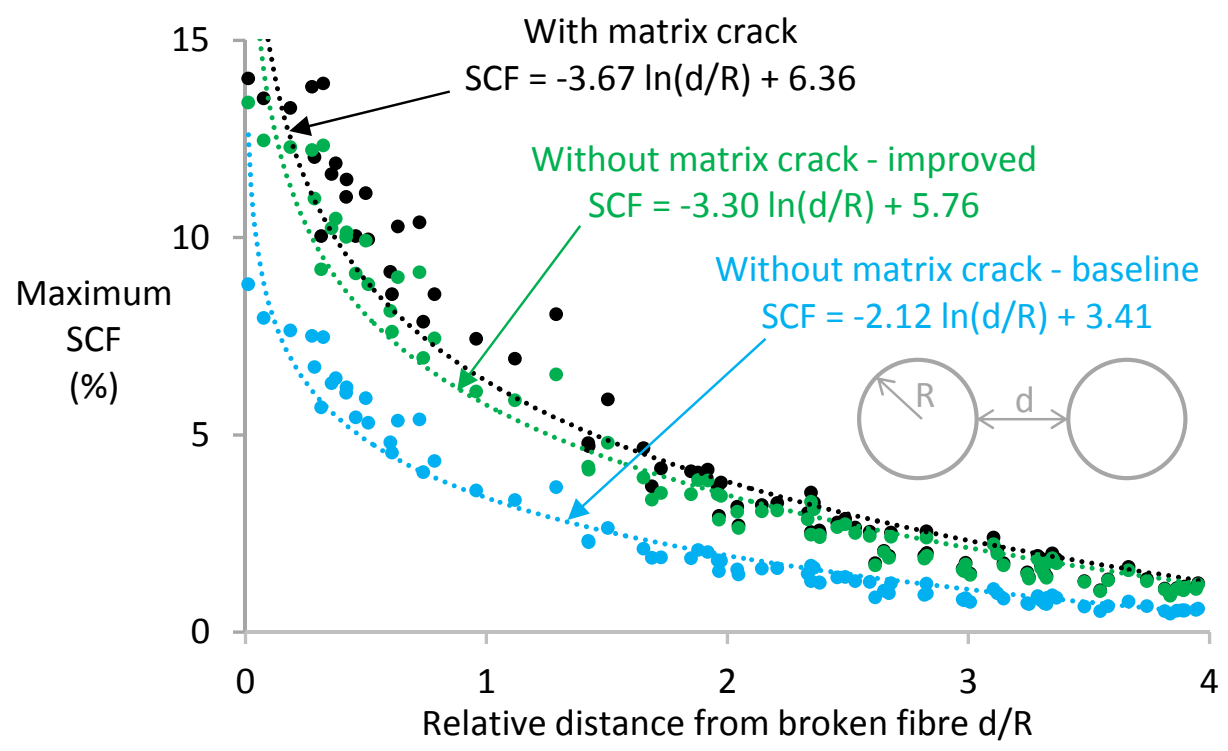

Figure 3: The maximum stress concentration factors in intact fibres around a single fibre break as a function of the relative distance from the broken fibre. The stress concentration factor is shown as the percentage by which it exceeds unity. Each data point represents one intact fibre in one of the 5 realisations of the FE model. 


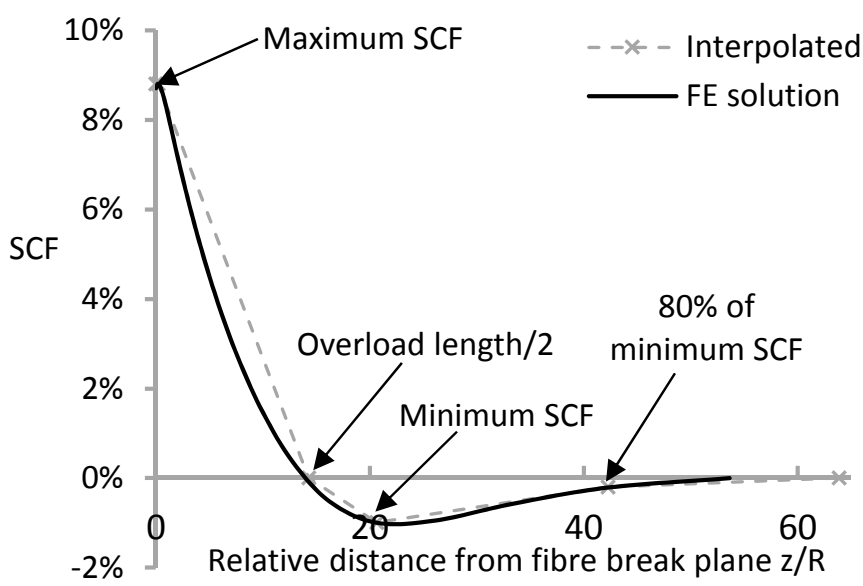

Figure 4: Example of the four reference points for the stress concentration factor profile in intact fibres adjacent to a fibre break. Piecewise linear interpolation is used in between these points. The stress concentration factor is shown as the percentage by which it exceeds unity.

\subsection{Interacting breaks}

The previous section only describes the stress redistribution around isolated fibre breaks. To properly predict final failure, however, the interactions among fibre breaks must be taken into account. Influence superposition rules have been developed to predict these stress redistribution around interacting fibre breaks based on shear lag solutions for a single break [21,34,38]. We sought out an approximate approach that fits better in the framework of the present model. Linear superposition of the single fibre break solutions would fit in this framework. An important downside, however, is that it neglects SCFs that the fibre breaks exert on each other. That neglect leads to predictions that do not maintain force equilibrium.

In view of this, an enhanced superposition principle is developed that does maintain force equilibrium. This principle first applies linear superposition to single break solutions, but then additionally distributes the SCFs that the fibre breaks exert on each other. This redistribution is performed proportional to the SCFs obtained from linear superposition. This procedure is further clarified in Fig. 5 for a hexagonal packing where the SCFs are concentrated on the nearest neighbours only. For the linear superposition in Fig. 5a, an SCF of 33\% is missing. This SCF has been redistributed proportionally in Fig. 5b. Note that a larger portion of this stress redistribution ends up on the two fibres that previously carried $33 \%$.

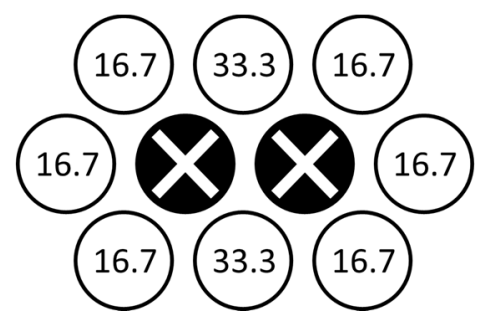

(a)

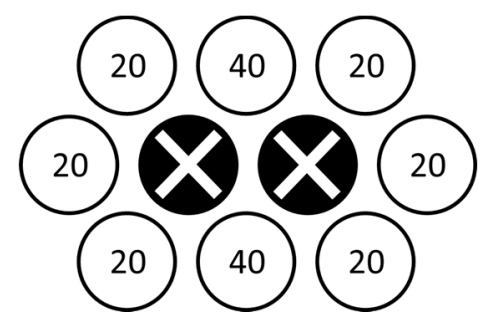

(b)

Figure 5: Illustration of the superposition principles in the fibre break plane: (a) linear superposition, and (b) enhanced superposition. The white crosses indicate fibre breaks. A hexagonal packing is assumed to simplify the situation, and the SCFs are assumed to be concentrated on the nearest neighbours only. 
A set of $4 \mathrm{FE}$ models was created to analyse the stress redistribution around three fibre breaks and validate the enhanced superposition principle. Fig. 6 illustrates these 4 models, which all have exactly the same random packing realisation and mesh. Three models with single fibre breaks and one with all three fibres broken were obtained by changing the boundary conditions. Linear and enhanced superposition results are computed based on the individual fibre break solutions. Their relative error in the maximum SCF is computed by comparison of the FE model with three fibre breaks.

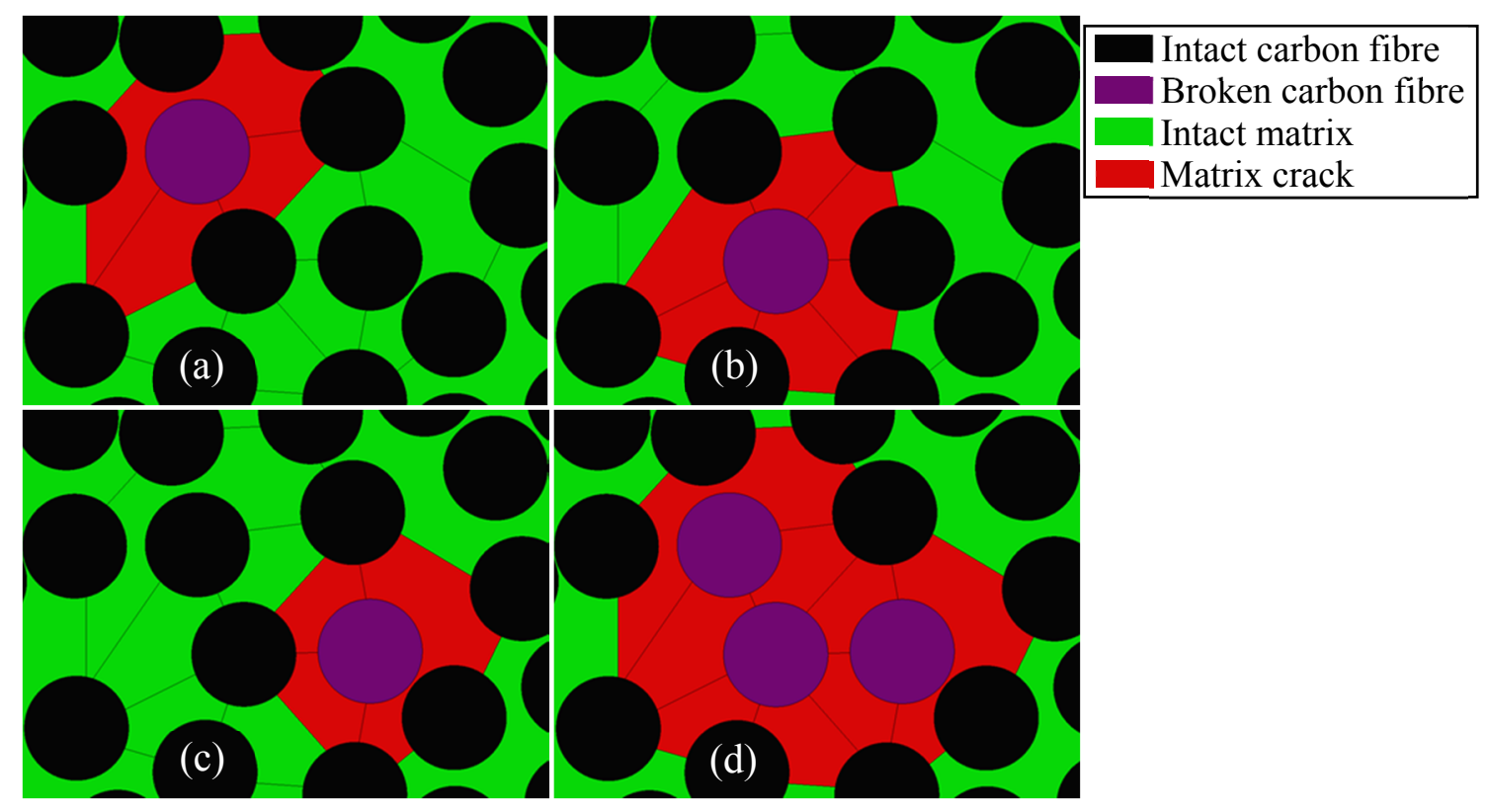

Figure 6: Illustration of the 4 FE models in the fibre break plane: (a) fibre break \#1, (b) fibre break \#2, (c) fibre break \#3, and (d) all three coplanar fibre breaks. For models with matrix cracks, the symmetry condition on the matrix crack region (red) is replaced by traction free conditions.

Fig. 7 plots the relative errors from both superposition principles compared to the FE solution with all 3 fibre breaks. If the superposition principle and full FE solution predict an SCF of $6 \%$ and $5 \%$ respectively, then the relative error would be $+20 \%$. Fig. 7 shows that both superposition principles lead to significant errors if no matrix crack is present. Both superposition principles have an average error of $7 \%$ in this case. These errors are attributed to the complex stress field at the perimeter of the fibre breaks, which is altered by the presence of the other fibre breaks. In case of matrix cracks or the improved strategy without matrix cracks, however, this complex stress field is avoided in the broken fibre, where its effect on the SCFs is smaller. Fig. 7 shows that the enhanced superposition principle is more reliable than linear superposition in both cases. The average error for the enhanced superposition principle reduces to $-1 \%$ and $+2 \%$ for matrix cracks and the improved strategy without matrix cracks, respectively. This shows that this relatively simple superposition principle successfully predicts SCFs around multiple fibre breaks. 


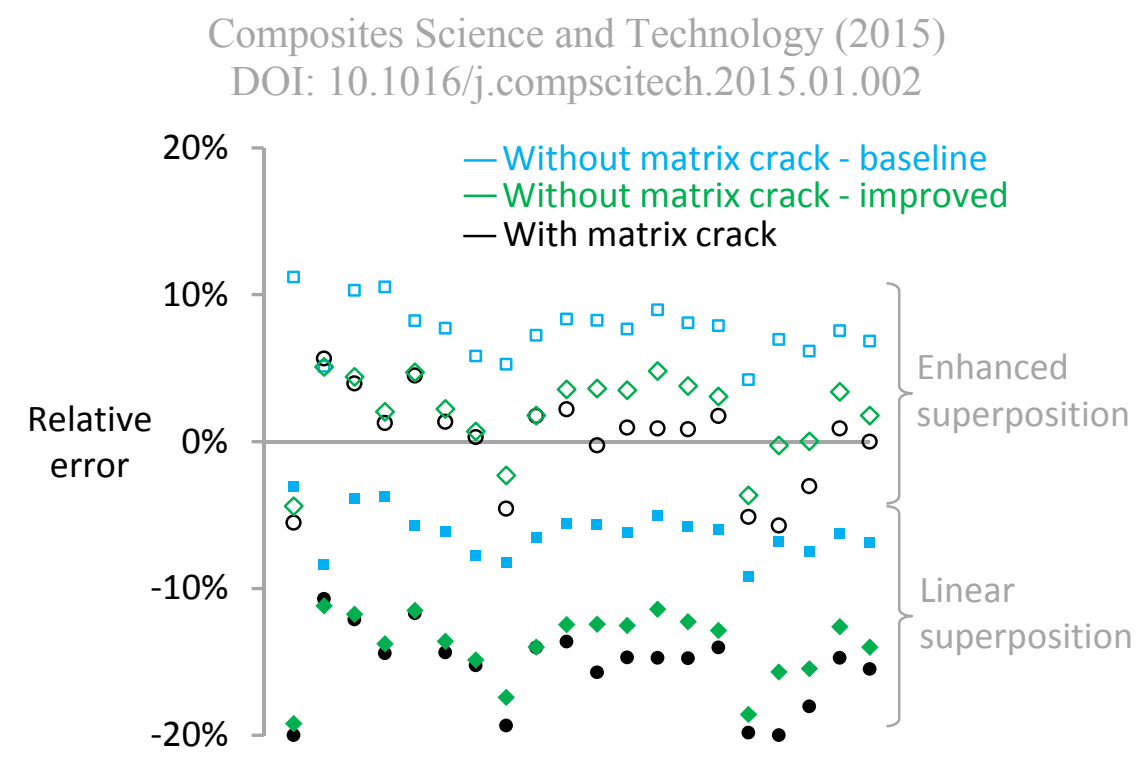

Figure 7: The relative error from both superposition principles based on 3 individual coplanar fibre breaks compared to the FE solution with 3 coplanar fibre breaks. The horizontal axis is arbitrary to allow depiction of the affected fibres and involves 20 fibres close to the fibre breaks.

Matrix cracks and the improved strategy shift the stress singularity into the matrix and avoids it in the broken fibre. It is hypothesised that this leads to better SCF predictions. The remainder of this paper therefore uses the enhanced superposition principle. It was verified in the strength model that the overall force was the same on all planes.

FE models with multiple fibre breaks confirmed that the ineffective length increases for larger clusters. At present, this is not implemented in the strength model. The interactions between fibre breaks are hence taken into account for intact fibres, but not for the ineffective length of the broken fibres.

\section{Strength model}

\subsection{General approach}

A strength model for UD composites has been devised, with the aim of increased versatility compared to existing models. Fig. 8 illustrates the flow chart for the strength model. The model is a Monte Carlo simulation based on Rosen's chain-of-bundles approach [39]. The general modelling approach is based on the works of Curtin, Okabe and co-workers $[6-8,12,17,18,40]$. A representative volume element (RVE) is created, consisting of parallel fibres in a random fibre packing. These packings have a 50\% fibre volume fraction and were created using the generator described in [41,42]. The cylindrical RVE contains 10,000 fibres plus an additional 530 fibres on its perimeter. These boundary fibres can carry SCFs, but are not allowed to break. They are also not taken into account in the calculation of the average composite stress. The fewer nearby fibres at the perimeter would cause increased SCFs, and hence preferential cluster formation at the perimeter. The boundary fibres avoid this, making the model more representative of larger RVE sizes. In larger models, the relative importance of the perimeter is smaller and cluster development is less likely to start in that region. Every fibre is split up in 2857 cylindrical elements, each with a length of $3.5 \mu \mathrm{m}$, resulting in a total model length of $10 \mathrm{~mm}$. 


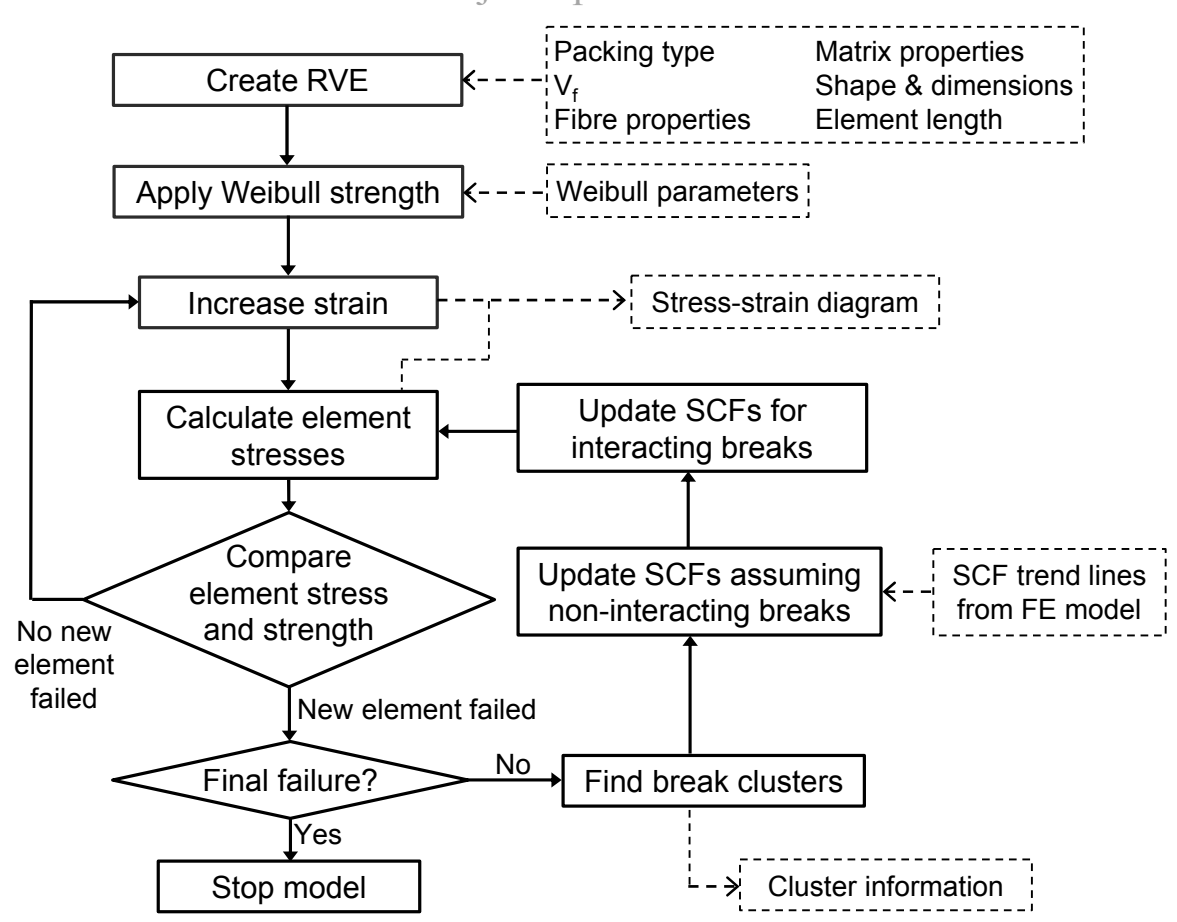

Figure 8: Flow chart for the strength model. The dashed rectangles indicate the inputs and outputs.

The material parameters are based on a T700 carbon fibre in an epoxy matrix. The strength model only uses the longitudinal fibre tensile modulus, while the FE model used the entire set of engineering constants. The engineering constants of this carbon fibre with a $230 \mathrm{GPa}$ longitudinal tensile modulus are described in [13]. The epoxy matrix is assumed to be elasto-plastic with a tensile modulus of $2.95 \mathrm{GPa}$ and a Poisson's ratio of 0.4. The stress-strain diagram of the matrix was taken from Okabe et al. [6], as this was found to be a reliable source.

A strength consistent with a Weibull distribution is assigned to each fibre element. Various Weibull distributions for T700 carbon fibres have been examined. The data set of Watanabe et al. [43] is found to be the most reliable, as it is based on extensive experiments. This data set is based on a bimodal Weibull distribution $P$, which assumes that carbon fibres contain two types of strength-determining flaws such that:

$$
P\left(\sigma_{f}\right)=1-\exp \left[-\frac{L}{L_{0}} \cdot\left(\frac{\sigma_{f}}{\sigma_{01}}\right)^{m_{1}}-\frac{L}{L_{0}} \cdot\left(\frac{\sigma_{f}}{\sigma_{02}}\right)^{m_{2}}\right]
$$

in which $\sigma_{f}$ is the fibre strength, $\sigma_{01}$ and $\sigma_{02}$ are the Weibull scale parameters for the first and second flaw distribution, respectively, $L$ is the element length, $L_{0}$ is the reference gauge length, and $m_{1}$ and $m_{2}$ are the Weibull moduli for the first and second flaw distribution respectively. These parameters are: $\sigma_{01}=5200 \mathrm{MPa}, \sigma_{02}=6100 \mathrm{MPa}$, $L_{0}=10 \mathrm{~mm}, m_{1}=4.8$ and $m_{2}=12.0$.

The strain is gradually incremented and element stresses are calculated, see Fig. 8. The stress $\sigma$ in each element is calculated as: $\sigma=\varepsilon \cdot E \cdot(1+S C F / 100 \%)$, where $\varepsilon$ is the global axial strain, $E$ the Young's modulus of the fibre, and $S C F$ the stress concentration factor as the percentage by which it exceeds unity. The $S C F$ values are 
extracted from the stress profiles in FE models with a single fibre break. These FE models use the same type of random packings and fibre volume fraction, but contain fewer and shorter fibres to keep the model tractable. The FE results are imported into the strength model by fitting trend lines through the data points, as was illustrated in Fig. 3. This allows the use of accurate stress profiles in a computationally tractable manner. Changes in element length and position due to the applied SCFs are not taken into account, causing the predicted stress-strain diagrams to be linear.

Failure of each element is checked by comparing the element stress with its strength. If new elements have failed, then the model checks for final composite failure. Calculations are terminated if at least $10 \%$ of the fibres have broken within an axial segment of $35 \mu \mathrm{m}$. This length corresponds to the length over which the SCFs are significantly increased by the fibre break. The criterion is chosen as it corresponds to an exponential increase in the number of fibre breaks within the same strain increment. Such rapid increase in fibre failure is associated with final composite failure.

If the composite failure criterion is not satisfied and a new fibre element has failed, then the model updates the break clusters. Two fibre breaks are considered to be part of the same break cluster if: (1) the lateral distance between the fibre centres is smaller than 4 fibre radii, and (2) the axial distance between them is less than 30 fibre radii. These criteria correspond to the region where the SCFs are significant. The model then updates the SCFs assuming that they do not interact with each other. In the next step, these interactions are taken into account.

Since the application of SCFs changes element stresses, these stresses are updated and compared to their strength again. The SCFs and stresses are updated until no new element fails. Then, the strain is further incremented and the procedure is repeated until final composite failure is detected.

\subsection{Failure strain}

Fig. 9 reveals a predicted failure strain of $2.84 \%$ for baseline models without matrix cracks. This reduces to $2.40 \%$ for the improved strategy and $2.38 \%$ in case of matrix cracks. This confirms the importance of finding the right boundary conditions to model a single fibre break, as they have a major influence on the failure strain predictions. Furthermore, comparing the matrix crack results to the improved strategy without matrix cracks reveals that matrix cracks hardly affect the failure strain predictions. This could be expected based on the results in Fig. 2 and Fig. 3.

Even with matrix cracks, the model still overestimates the failure strain by $0.28 \%$ compared to the data sheet value of $2.1 \%$ for this carbon fibre/epoxy [44]. This discrepancy is attributed to (1) the small model size and (2) the neglect of the non-linear elastic behaviour of carbon fibre [45]. The latter effect alone would reduce the predicted failure strain by roughly $20 \%$, while maintaining the same predicted strength. 


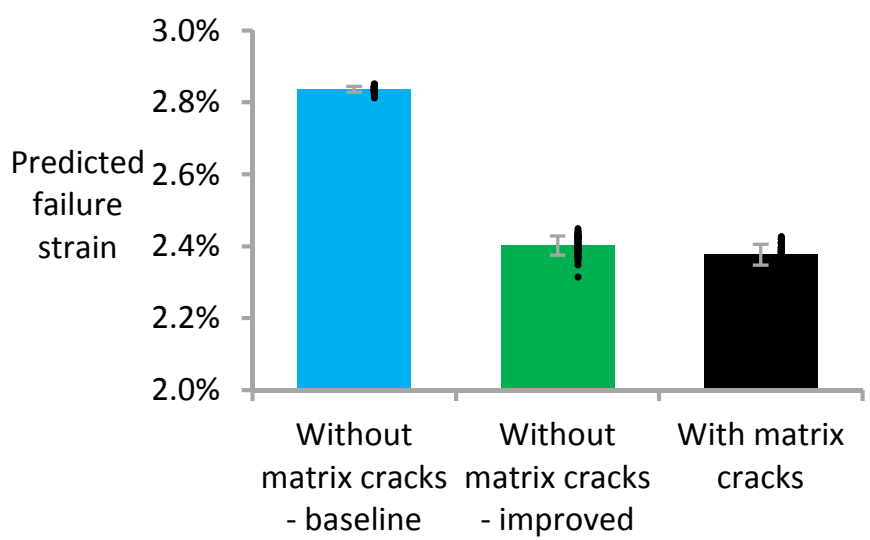

Figure 9: Predicted failure strain for 50 realisations of the strength model. The error bars represent the standard deviation, while the dots represent the individual results for each realisation.

\subsection{Cluster development}

The internal damage development is now analysed to further understand the influence of matrix cracks. This analysis compares the cluster development without and with matrix cracks. For larger and more realistic model sizes, the results may be different but the comparison should remain the same. Fig. 10 plots the number of fibre breaks in clusters up to 5-plets. An "i-plet" is a cluster where "i" is the number of fibre breaks. The development of 1-plets is almost identical for both models, as this is controlled by the Weibull strength distribution instead of by SCFs. Fig. 10b demonstrates that 2-plets develop slightly earlier in models with matrix cracks. This difference is more pronounced for large break clusters. The higher SCFs and larger ineffective lengths associated with the presence of matrix cracks clearly cause a stronger tendency for development of break clusters. Models with matrix cracks yield nearly the same results as improved models without matrix cracks, which is expected as they had nearly the same failure strain (see Fig. 9). 


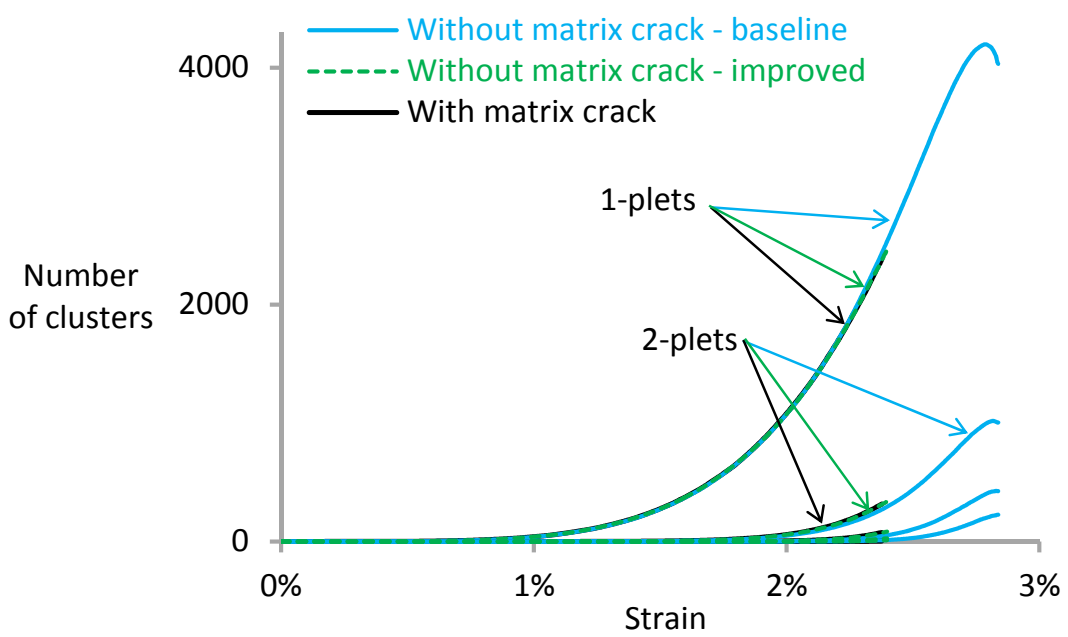

(a)

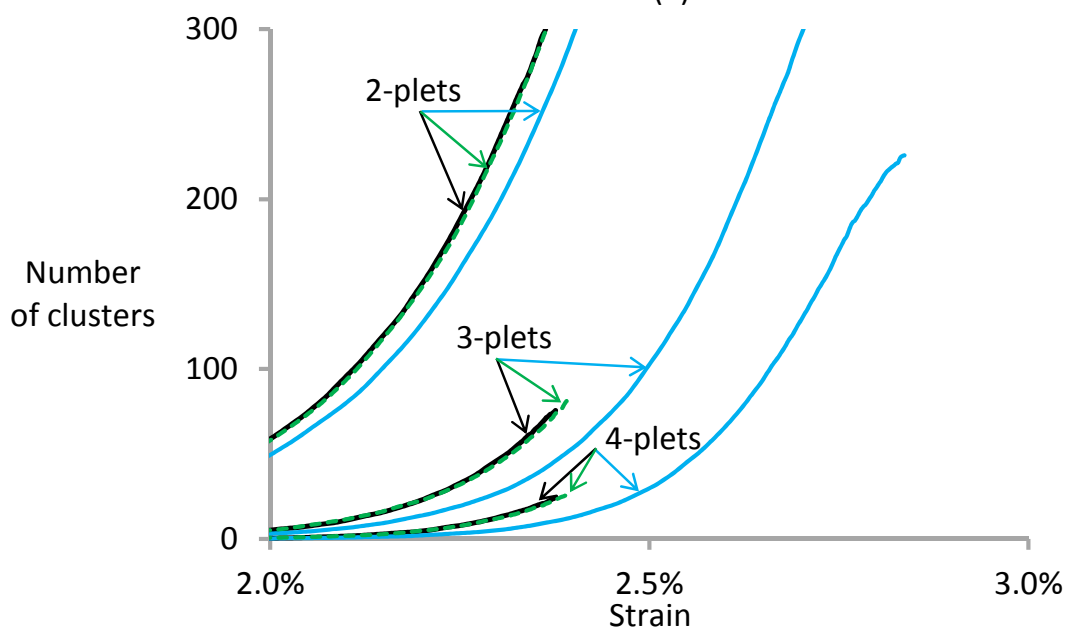

(b)

Figure 10: Break cluster development: (a) overview from zero to $3 \%$ strain, and (b) more detailed view at high applied strains.

Fig. 11 plots the critical cluster size, which is the size is calculated as the largest cluster in the last strain increment of the calculation just before final failure. The average value of 360 for the baseline solution is an order of magnitude larger than the value of 24 for the improved solution. The improved solution without matrix cracks yields an average value close to the 21 found in the presence of matrix cracks. This small difference stems from the slightly higher SCFs in models with matrix cracks (see Fig. 3). Comparing these values to experimental data is, however, challenging due to differences in the cluster definition. 


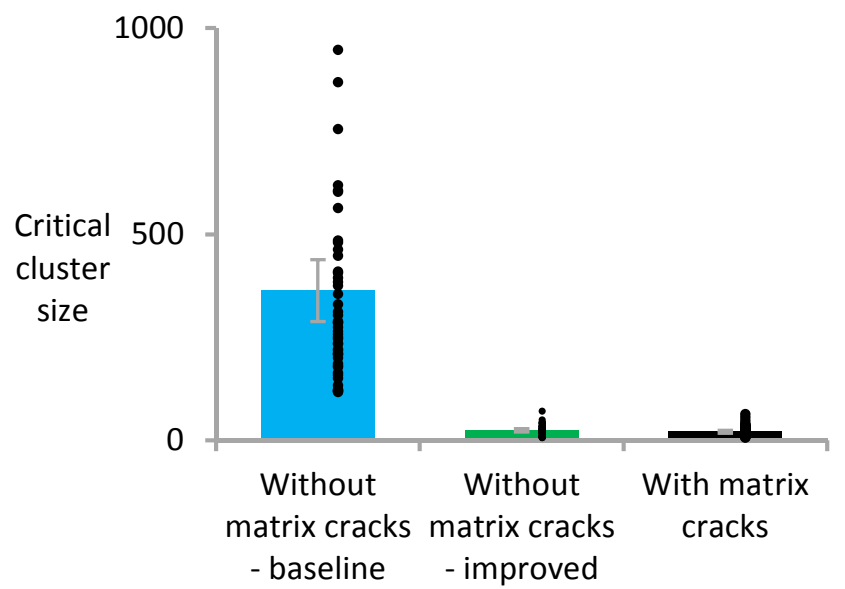

Figure 11: The critical cluster size for 50 realisations of the strength model. The individual results are plotted together with the $\mathbf{9 5 \%}$ confidence intervals.

Finally, Fig. 12 depicts the largest break cluster as a function of applied strain. Up to $2.3 \%$ strain, the largest clusters are all similar. At higher strains, the largest cluster rapidly increases in size for the models with matrix cracks and improved models without matrix cracks. This process is slower for baseline models without matrix cracks, and only speeds up at higher applied strains.

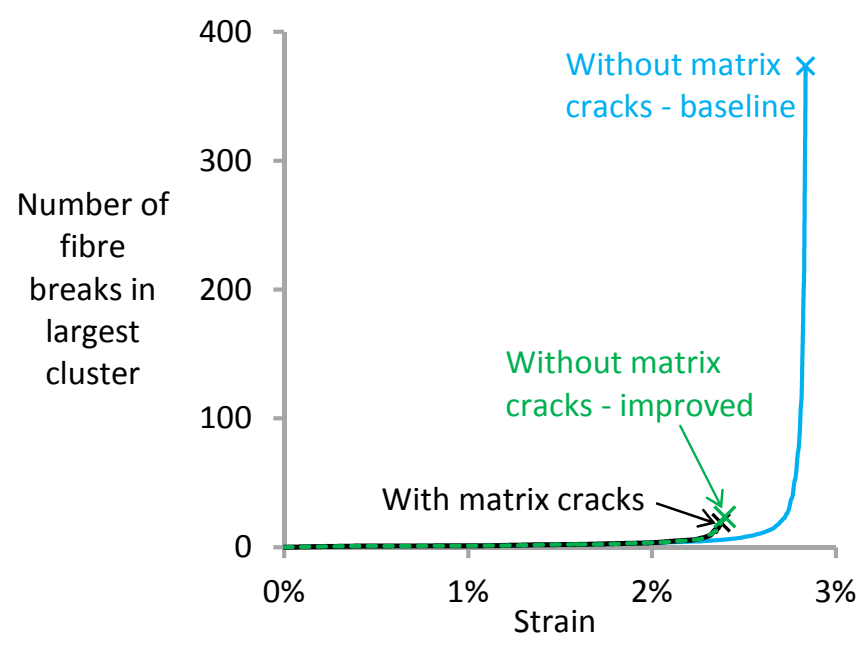

Figure 12: Evolution of the largest cluster of fibre breaks as a function of the applied strain. The crosses indicate the average composite failure strain.

\section{Conclusion}

A model was presented with increased versatility compared to state-of-the-art methods. This model is capable of incorporating various matrix and fibre phenomena, but the present study focused on the effects of matrix cracks around fibre breaks. Compared to the improved strategy without matrix cracks, the presence of matrix cracks was found to have little influence on the SCFs and ineffective length. By incorporating these results into a strength model, the negligible influence of matrix cracks on the cluster development and failure strain was confirmed. Furthermore, a simplified superposition principle was proposed and shown to be reliable for predicting SCFs around multiple fibre breaks. 
The presented model assumes that matrix cracks occur around fibre breaks to relieve what would otherwise be infinite stress concentrations. More experimental work is needed to establish if and when this matrix cracking occurs. Alternatively, the matrix could yield or the fibre-matrix interface could debond. In-depth experimental work is needed to clarify under which conditions these three phenomena occur. The model is being further developed to predict all three possible scenarios and combinations thereof. These results will be reported in future publications, and will be crucial in the in-depth experimental validation that is currently ongoing.

\section{Acknowledgements}

The work leading to this publication has received funding from the European Union Seventh Framework Programme (FP7/2007-2013) under the topic NMP-2009-2.5-1, as part of the project HIVOCOMP (Grant Agreement No. 246389). The authors thank the Agency for Innovation by Science and Technology in Flanders (IWT) for a PhD. grant and FWO Flanders for a travel grant to Y. Swolfs. The authors also thank A.R. Melro and P. Camanho for the permission to use their random fibre packing generator. I. Verpoest holds the Toray Chair in Composite Materials at KU Leuven. We acknowledge support from the UCSB Center for Scientific Computing at CNSI, the UCSB MRL: an NSF MRSEC (DMR-1121053) and NSF Grant CNS-0960316.

\section{References}

[1] Harlow DG, Phoenix SL. The chain-of-bundles probability model for the strength of fibrous materials I: Analysis and conjectures. Journal of Composite Materials. 1978;12(APR):195-214.

[2] Phoenix SL, Smith RL. A comparison of probabilistic techniques for the strength of fibrous materials under local load-sharing among fibers. International Journal of Solids and Structures. 1983;19(6):479-496.

[3] Smith RL, Phoenix SL, Greenfield MR, Henstenburg RB, Pitt RE. Lower-tail approximations for the probability of failure of three-dimensional fibrous composites with hexagonal geometry Proceedings of the Royal Society of London Series A: Mathematical Physical and Engineering Sciences. 1983;388(1795):353-391.

[4] Scott AE, Sinclair I, Spearing SM, Thionnet A, Bunsell AR. Damage accumulation in a carbon/epoxy composite: Comparison between a multiscale model and computed tomography experimental results. Composites Part A: Applied Science and Manufacturing. 2012;43(9):1514-1522.

[5] Wada A, Fukuda H. Approximate upper and lower bounds for the strength of unidirectional composites. Composites Science and Technology. 1999;59(1):89-95.

[6] Okabe T, Ishii K, Nishikawa M, Takeda N. Prediction of Tensile Strength of Unidirectional CFRP Composites. Advanced Composite Materials. 2010;19(3):229-241.

[7] Okabe T, Takeda N. Size effect on tensile strength of unidirectional CFRP composites - experiment and simulation. Composites Science and Technology. 2002;62(15):2053-2064.

[8] Noda J, Okabe T, Takeda N, Shimizu M. Tensile strength of CFRP cross-ply laminates containing transverse cracks. Advanced Composite Materials. 2006;15(1):8193. 
[9] Hedgepeth JM. Stress concentrations in filamentary structures. NASA TN. 1961;D882( ):1-36.

[10] Fukuda H. Stress concentration factors in unidirectional composites with random fiber spacing. Composites Science and Technology. 1985;22(2):153-163.

[11] Xia Z, Curtin WA, Okabe T. Green's function vs. shear-lag models of damage and failure in fiber composites. Composites Science and Technology. 2002;62(10-11):12791288.

[12] Xia Z, Okabe T, Curtin WA. Shear-lag versus finite element models for stress transfer in fiber-reinforced composites. Composites Science and Technology. 2002;62(9):1141-1149.

[13] Swolfs Y, Gorbatikh L, Romanov V, Orlova S, Lomov SV, Verpoest I. Stress concentrations in an impregnated fibre bundle with random fibre packing. Composites Science and Technology. 2013;74:113-120.

[14] Swolfs Y, Gorbatikh L, Verpoest I. Stress concentrations in hybrid unidirectional fibre-reinforced composites with random fibre packings. Composites Science and Technology. 2013;85:10-16.

[15] Nedele MR, Wisnom MR. Three-dimensional finite element analysis of the stress concentration at a single fibre break. Composites Science and Technology. 1994;51(4):517-524.

[16] Behzadi S, Jones FR. The effect of temperature on stress transfer between a broken fibre and the adjacent fibres in unidirectional fibre composites. Composites Science and Technology. 2008;68(13):2690-2696.

[17] Xia Z, Curtin WA, Peters PWM. Multiscale modeling of failure in metal matrix composites. Acta Materialia. 2001;49(2):273-287.

[18] Xia ZH, Curtin WA. Multiscale modeling of damage and failure in aluminummatrix composites. Composites Science and Technology. 2001;61(15):2247-2257.

[19] de Morais AB. Stress distribution along broken fibres in polymer-matrix composites. Composites Science and Technology. 2001;61(11):1571-1580.

[20] Landis CM, McMeeking RM. A shear-lag model for a broken fiber embedded in a composite with a ductile matrix. Composites Science and Technology. 1999;59(3):447457.

[21] Beyerlein IJ, Phoenix SL. Stress concentrations around multiple fiber breaks in an elastic matrix with local yielding or debonding using quadratic influence superposition. Journal of the Mechanics and Physics of Solids. 1996;44(12):1997-2039.

[22] Blassiau S, Thionnet A, Bunsell AR. Micromechanisms of load transfer in a unidirectional carbon fibre-reinforced epoxy composite due to fibre failures. Part 2: Influence of viscoelastic and plastic matrices on the mechanisms of load transfer. Composite Structures. 2006;74(3):319-331.

[23] Smith RL. The random variation of stress concentration factors in fibrous composites. Journal of Materials Science Letters. 1983;2(8):385-387.

[24] Landis CM, McMeeking RM. Stress concentrations in composites with interface sliding, matrix stiffness and uneven fiber spacing using shear lag theory. International Journal of Solids and Structures. 1999;36(28):4333-4361. 
[25] Swolfs Y, Verpoest I, Gorbatikh L. Issues in strength models for unidirectional fibre-reinforced composites. Composites Science and Technology, accepted. 2015.

[26] Behzadi S, Curtis PT, Jones FR. Improving the prediction of tensile failure in unidirectional fibre composites by introducing matrix shear yielding. Composites Science and Technology. 2009;69(14):2421-2427.

[27] Swolfs Y, McMeeking RM, Gorbatikh L, Verpoest I. The effect of fibre dispersion on initial failure strain and cluster development in unidirectional carbon/glass hybrid composites. Composites Part A: Applied Science and Manufacturing. 2015;69:279-287.

[28] Ohno N, Okabe S, Okabe T. Stress concentrations near a fiber break in unidirectional composites with interfacial slip and matrix yielding. International Journal of Solids and Structures. 2004;41(16-17):4263-4277.

[29] Zhao FM, Takeda N. Effect of interfacial adhesion and statistical fiber strength on tensile strength of unidirectional glass fiber/epoxy composites. Part I: experiment results. Composites Part A-Applied Science and Manufacturing. 2000;31(11):12031214.

[30] Pegoretti A, Accorsi ML, Dibenedetto AT. Fracture toughness of the fibre-matrix interface in glass-epoxy composites. Journal of Materials Science. 1996;31(23):61456153.

[31] Johnson AC, Zhao FM, Hayes SA, Jones FR. Influence of a matrix crack on stress transfer to an alpha-alumina fibre in epoxy resin using FEA and photoelasticity. Composites Science and Technology. 2006;66(13):2023-2029.

[32] Netravali AN, Henstenburg RB, Phoenix SL, Schwartz P. Interfacial shear strength studies using the single-filament-composite test. 1. Experiments on graphite fibers in epoxy. Polymer Composites. 1989;10(4):226-241.

[33] Chateau C, Gelebart L, Bornert M, Crepin J, Caldemaison D, Sauder C. Modeling of damage in unidirectional ceramic matrix composites and multi-scale experimental validation on third generation $\mathrm{SiC} / \mathrm{SiC}$ minicomposites. Journal of the Mechanics and Physics of Solids. 2014;63:298-319.

[34] Li H, Jia Y, Mamtimin G, Jiang W, An L. Stress transfer and damage evolution simulations of fiber-reinforced polymer-matrix composites. Materials Science and Engineering A. 2006;425(1-2):178-184.

[35] Mishnaevsky Jr L, Brøndsted P. Micromechanisms of damage in unidirectional fiber reinforced composites: 3D computational analysis. Composites Science and Technology. 2009;69(7-8):1036-1044.

[36] Fiedler B, Klisch A, Schulte K. Stress concentrations in multiple fibre model composites. Composites Part A: Applied Science and Manufacturing. 1998;29(910):1013-1019.

[37] van den Heuvel PWJ, Peijs T, Young RJ. Failure phenomena in two-dimensional multi-fibre microcomposites. Part 4: a Raman spectroscopic study on the influence of the matrix yield stress on stress concentrations. Composites Part A: Applied Science and Manufacturing. 2000;31(2):165-171.

[38] Sastry AM, Phoenix SL. Load redistribution near non-aligned fiber breaks in a 2dimensional unidirectional composite using break-influence superposition. Journal of Materials Science Letters. 1993;12(20):1596-1599. 
[39] Rosen BW. Tensile failure of fibrous composites. AIAA Journal. 1964;2(11):19851991.

[40] Zhang F, Lisle T, Curtin WA, Xia Z. Multiscale modeling of ductile-fiberreinforced composites. Composites Science and Technology. 2009;69(11-12):18871895.

[41] Melro AR, Camanho PP, Pinho ST. Generation of random distribution of fibres in long-fibre reinforced composites. Composites Science and Technology. 2008;68(9):2092-2102.

[42] Romanov V, Lomov SV, Swolfs Y, Orlova S, Gorbatikh L, Verpoest I. Statistical analysis of real and simulated fibre arrangements in unidirectional composites. Composites Science and Technology. 2013;87:126-134.

[43] Watanabe J, Tanaka F, Okabe T. The tensile strength distribution of carbon fibers at short gauge length. 38th Conference of the Japan Society for Composite Materials, 2013. p. 171-172.

[44] T700S carbon fibre data sheet, www.toraycfa.com, June 2014.

[45] Toyama N, Takatsubo J. An investigation of non-linear elastic behavior of CFRP laminates and strain measurement using Lamb waves. Composites Science and Technology. 2004;64(16):2509-2516. 